\title{
Assessing the impact of air pollutants on clinical visits for childhood allergic respiratory disease induced by house dust mite in Shanghai, China
}

Junyang $\mathrm{Li}^{1+}$, Yabin $\mathrm{Hu}^{2+}{ }^{2}$, Huaiyuan $\mathrm{Li}^{3}$, Yihang Lin ${ }^{1}$, Shilu Tong ${ }^{2,4,5,6^{*}}$ and Youjin $\mathrm{Li}^{1^{*}}$ (1)

\begin{abstract}
Background: The prevalence of allergic respiratory disease (ARD) is increasing worldwide during the last few decades, causing a great disease burden especially for children. Air pollution has been increasingly considered as a potential contributor to this trend, but its role in ARD induced by house dust mite (HDM-ARD) remains unclear, especially in time-series study.
\end{abstract}

Methods: A positive reporting of respiratory allergy to named allergens was included by serum specific lgE testing. A time series Quasi-Poisson regression with distributed lag non-linear model, combined with generalized linear model was used to examine the effects of air pollutants on ARD, HDM-ARD and ARD induced by non-house dust mite (NHDM-ARD).

Results: A total of 16,249 cases of ARD, including 8,719 HDM-ARD and 8,070 NHDM-ARD from 1 Jan 2013 to 31 Dec 2017 were involved in this study. Air pollutants were significantly associated with clinical visits for childhood ARD and $\mathrm{HDM}$-ARD. Exposure to higher $\mathrm{O}_{3}$ and interquartile range (IQR) increment in $\mathrm{O}_{3}\left(40.6 \mathrm{\mu g} / \mathrm{m}^{3}\right)$ increased the risks

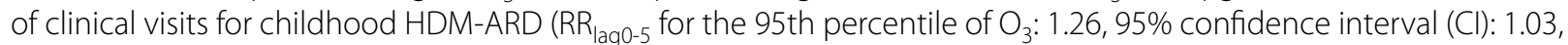
1.55; $\mathrm{RR}_{\text {lag0-5 }}$ for IQR increment $\left.\left(40.6 \mathrm{\mu g} / \mathrm{m}^{3}\right): 1.09,95 \% \mathrm{Cl}: 1.01,1.17\right)$ and $A R D\left(\mathrm{RR}_{\text {lag0-5 }}\right.$ for the 95th percentile of $\mathrm{O}_{3}$ : $1.19,95 \% \mathrm{Cl}: 1.03,1.38 ; \mathrm{RR}_{\text {lag0-5 }}$ for IQR increment $\left.\left(40.6 \mu \mathrm{g} / \mathrm{m}^{3}\right): 1.06,95 \% \mathrm{Cl}: 1.01,1.12\right)$. In addition, higher $\mathrm{O}_{3}$ was associated with increased $R R$ of boys with ARD ( $R_{\text {lag0-5 }}$ for the 95th percentile: $1.26,95 \%$ Cl: 1.05, 1.51; RR lag0-5 $_{\text {for IQR }}$ increment $\left.\left(40.6 \mu \mathrm{g} / \mathrm{m}^{3}\right): 1.09,95 \% \mathrm{Cl}: 1.02,1.16\right)$ and HDM-ARD (RR lag0-5 $_{\text {for }}$ fhe 95 th percentile: $1.36,95 \% \mathrm{Cl}: 1.06,1.75$; $\mathrm{RR}_{\text {lag0-5 }}$ for IQR increment $\left.\left(40.6 \mu \mathrm{g} / \mathrm{m}^{3}\right): 1.11,95 \% \mathrm{Cl}: 1.02,1.22\right)$, but not in girls.

Conclusions: Exposure to $\mathrm{O}_{3}$ appeared to be a trigger of clinical visits for childhood ARD, especially for HDM-ARD and boys. These findings provide novel evidence on the impact of air pollution on HDM-ARD, which may have

\footnotetext{
*Correspondence: tongshilu@scmc.com.cn; kevinliyoujin@outlook.com

†Junyang Li and Yabin Hu contributed equally to this work

1 Department of Otolaryngology, Shanghai Children's Medical Center,

School of Medicine, Shanghai Jiao Tong University, 1678 Dongfang Rd,

Pudong, Shanghai 200127, China

2 Department of Clinical Epidemiology and Biostatistics, Shanghai

Children's Medical Center, School of Medicine, Shanghai Jiao Tong

University, 1678 Dongfang Rd, Pudong, Shanghai 200127, China

Full list of author information is available at the end of the article
}

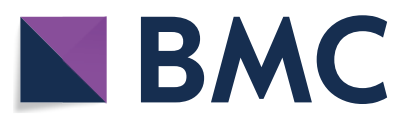

(c) The Author(s) 2022. Open Access This article is licensed under a Creative Commons Attribution 4.0 International License, which permits use, sharing, adaptation, distribution and reproduction in any medium or format, as long as you give appropriate credit to the original author(s) and the source, provide a link to the Creative Commons licence, and indicate if changes were made. The images or other third party material in this article are included in the article's Creative Commons licence, unless indicated otherwise in a credit line to the material. If material is not included in the article's Creative Commons licence and your intended use is not permitted by statutory regulation or exceeds the permitted use, you will need to obtain permission directly from the copyright holder. To view a copy of this licence, visit http://creativecommons.org/licenses/by/4.0/. The Creative Commons Public Domain Dedication waiver (http://creativeco mmons.org/publicdomain/zero/1.0/) applies to the data made available in this article, unless otherwise stated in a credit line to the data. 
significant implications for designing effective intervention programs to control and prevent childhood ARD, especially HDM-ARD, in China and other similar developing countries.

Keywords: Air pollutants, Allergic respiratory diseases, Allergic respiratory diseases induced by house dust mite, Children, Clinical visits

\section{Background}

Since the publication of the Allergic Rhinitis and its Impact on Asthma (ARIA) document in 2001 [1], the "one airway" concept has been accepted almost unanimously by the physicians to describe specific aspects of patients diagnosed with allergic rhinitis (AR) with or without allergic asthma (AA). The clinical phenotypes of $A R$ and AA relevant to allergy are encompassed in the term "allergic respiratory disease" (ARD) and the concept of a united allergic airway reflects a shared underlying mechanism of pathogenesis. The increasing prevalence of ARD has been assessed by many epidemiological studies worldwide [2-4]. Importantly, environmental factors have been increasingly considered as potential major contributors to this trend $[5,6]$.

Although the exact pathogenesis of ARD remains unclear, the increased presence of outdoor air pollutants resulting from more intense energy consumption and exhaust emissions from cars and other vehicles, may play an important role in the development of ARD [7]. Air pollutants have been reported to be associated with worsening of ARD symptoms [8]. Nevertheless, allergens play a decisive role in the onset of symptoms and influence the clinical manifestations of ARD [9]. House dust mite (HDM) sensitization is a major causative factor in the development of ARD [10]. Furthermore, a study has revealed that HDM induced more severe late reactions than cat or pollens in asthmatic patients [11]. Components of the ultrafine fraction of particulate matter (PM) induce allergic pulmonary inflammation and act as adjuvant of the allergic response to HDM [12], which suggests that airway mucosal damage and impaired mucociliary clearance induced by air pollutants may facilitate the access of inhaled HDM to the cells of the immune system.

To the best of our knowledge, no study has addressed the effects of air pollution on ARD induced by HDM (HDM-ARD) and/or non-house dust mite (NHDMARD) to date. Therefore, in this study, we investigated the independent effects of air pollutants on ARD, HDMARD, and NHDM-ARD in Shanghai, China.

\section{Methods}

\section{Study participants}

Shanghai, located in the east of China (N30 $40^{\prime}-31^{\circ} 53^{\prime}$, $\mathrm{E} 120^{\circ} 52^{\prime}-122^{\circ} 12^{\prime}$ ), is the most populous city in China, has a subtropical monsoon climate with four distinct seasons.
In this study, data on daily clinical visits (outpatient and inpatient visits) for childhood ARD, HDM-ARD and NHDM-ARD from 1 January 2013 to 31 December 2017 were collected from Shanghai Children's Medical Center, the largest pediatric hospital affiliated to Shanghai Jiao Tong University School of Medicine. The outpatient visits include allergic respiratory diseases such as allergic rhinitis and asthma, etc. The inpatient visits include emergence department and general admissions for respiratory disease. The medical record included the age, gender, date of visit, total IgE level, all types of inhaled allergens (including HDM (Dermatophagoides pteronyssinus [Der p 1] and Dermatophagoides farinae [Der f 1]), cat/dog hair, molds, cockroaches, grass/tree and pollens) detection and identification. Principal diagnostic classification of childhood ARD was made according to the tenth version of the International Classification of Diseases for anaphylaxis due to asthma (ICD-10, J45-J46) and AR (ICD-10, J30). The participants should meet the following criteria: at least one inhaled allergen was positive and total IgE was above the normal range. The exclusion criteria were as follows: (1) children were negative for allergens detection; or (2) total IgE was normal; or (3) patients older than 18 years old.

\section{Assessment of allergens-specific lgE and total lgE in serum}

Before testing, the performance qualification of total IgE had been done. The protocol had an inter-assay variance of $1.49 \%$ and an intra-assay variance of $1.63 \%$ when testing the low concentration $(70 \mathrm{IU} / \mathrm{mL})$ of quality control serum, and an inter-assay variance of $0.65 \%$ and an intra-assay variance of $1.65 \%$ when testing the high concentration $(200 \mathrm{IU} / \mathrm{mL})$ of quality control serum. Serum total IgE (TIgE) was determined quantitatively in all the patients using the BN II and BN ProSpec (N Latex IgE mono, Siemens, Germany), after calibration using commercial standard (also Siemens), and expressed in IU/ $\mathrm{mL}$. The TIgE reference range depends on the age of the individual [It ranges from 0 to $1.5 \mathrm{IU} / \mathrm{mL}$ in newborn infants, $0-15 \mathrm{IU} / \mathrm{mL}$ in infants during the first year of life, $0-60 \mathrm{IU} / \mathrm{mL}$ in children (1-5 years), $0-90 \mathrm{IU} / \mathrm{mL}$ in children (6-9 years), and $0-200 \mathrm{IU} / \mathrm{mL}$ in children $(10-$ 17 years)] [13]. HDM or HDM along with other more serum IgE species (sIgE) was found positive $(\geq 0.35 \mathrm{IU} /$ $\mathrm{ml}$ ) by Western blotting using AllergyScreen ${ }^{\mathrm{TM}}$ human serum specific IgE allergen detection kit for specific 
inhalant allergens as described in previous publications $[14,15]$. In this study, ARD induced by non-house dust mite such as cat/dog hair, molds, cockroaches, grass/tree and pollens was classified as NHDM-ARD.

\section{Air pollutants exposure assessment}

The data on air pollutants $\left(\mu \mathrm{g} / \mathrm{m}^{3}\right)$ were collected from the Shanghai Environmental Protection Agency, including nitrogen dioxide $\left(\mathrm{NO}_{2}\right)$, sulfur dioxide $\left(\mathrm{SO}_{2}\right)$, ozone $\left(\mathrm{O}_{3}\right)$, and airborne particulate matter with an aerodynamic diameter less than $2.5 \mu \mathrm{m}\left(\mathrm{PM}_{2.5}\right)$ or $10 \mu \mathrm{m}$ $\left(\mathrm{PM}_{10}\right)$. The meteorological data (daily mean temperature (Tmean, ${ }^{\circ} \mathrm{C}$ ) was obtained from the Shanghai Meteorological Service. Daily mean values of air pollutants and meteorological factors were calculated using the 24-h monitoring records. Daily averages of air pollutants from many monitoring stations in various regions of Shanghai were used in this study (Fig. 1). Shanghai Meteorological Service is located at the center of the city, providing monitoring meteorological data with well calibrated and highly related to the records in other stations $[16,17]$. In this study, we defined air pollutants as high level when the values were greater than the 95th percentile of each air pollutant.

\section{Statistical analysis}

The analysis of the data was conducted in three phases. Firstly, we did descriptive analysis using mean, standard deviation (SD), interquartile range (IQR), minimum, maximum, 25th percentile $\left(\mathrm{P}_{25}\right)$, 50th percentile $\left(\mathrm{P}_{50}\right)$ and 75 th percentile $\left(\mathrm{P}_{75}\right)$ to describe daily clinical visits and environmental factors. Spearman's correlation analysis was used to examine the correlations between

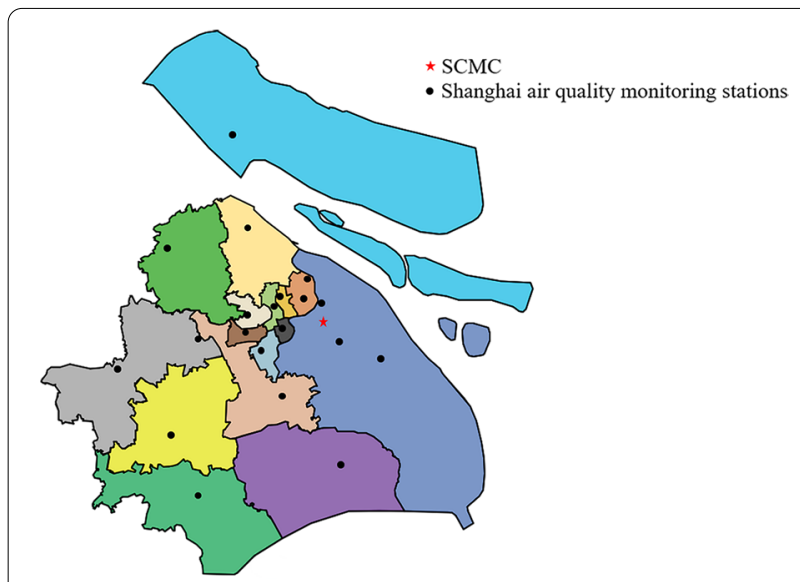

Fig. 1 The locations of pediatric hospital and air quality monitoring stations in this study. SCMC: Shanghai Children's Medical Center; Star represent the SCMC; Circles represent the monitoring stations environmental factors and clinical visits for childhood ARD, HDM-ARD and NHDM-ARD on the current day.

Secondly, a Quasi-Poisson generalized linear regression model combined with a distributed lag non-linear model (DLNM) [18] was used to determine the lagged and non-linear effects of air pollutants on childhood ARD, HDM-ARD and NHDM-ARD. Since Spearman correlation coefficients between $\mathrm{NO}_{2}, \mathrm{SO}_{2}, \mathrm{PM}_{10}$, and $\mathrm{PM}_{2.5}$ were high $\left(\mathrm{r}_{\mathrm{s}}>0.6\right)$, to avoid collinearity, only one of these variables was included in the final model, with the lowest Akaike information criterion (AIC) value. By comparing all multivariable models, we finally found that the model with Tmean and $\mathrm{O}_{3}$ performed the best, with the smallest AIC and residual deviance. The final multivariable model is as follows:

$$
\begin{aligned}
\text { Efficacy }(\%) \text { against ticks }= & 100 \times(\text { Mean count control } \\
& - \text { Mean count treated }) \\
& / \text { Mean count control. }
\end{aligned}
$$

where $E\left(\mathrm{Y}_{t}\right)$ is the number of daily clinical visits for childhood ARD, HDM-ARD or NHDM-ARD expected on day $t$; maxlag was set at 28 for temperature and 5 for $\mathrm{O}_{3}$ according to previous studies and the reference of AIC [19]; $\alpha$ is the intercept; day of the week (dow) and public holiday are controlled for as categorical variables; $n s($ time,$d f /$ year) is the natural cubic spline function (ns) for time (i.e., $1-1826$ in total), with $9 \mathrm{df} /$ year selected for the final model by calculating the minimum of the residuals using the partial autocorrelation function and based on the lowest AIC $[20,21]$; ns (time, df/year) was used to control for seasonality and long-term trends in childhood ARD; Tmean indicates the mean temperature. $c b$ represents the "cross-basis" function which defined the matrix about temp, $\mathrm{O}_{3}$ and lag using ns or linear function as appropriate.

Finally, sensitivity analyses were conducted to verify the robustness of the final results. We used 6-14 $d f$ per year for calendar time and 2-7 $d f$ for environmental factors in the model. The model included Tmean, $\mathrm{PM}_{2.5}$, and $\mathrm{O}_{3}$ were performed. We also set the maximum lag as 14 or 21 in the model to compare the results. We also did many subgroup analyses stratified by gender.

All analyses were conducted with $\mathrm{R}$ software 3.6.3. The statistical significance level was set at $\mathrm{p}$-value $<0.05$ (two-side).

\section{Ethics issue}

The ethical approval of this project was granted by the Ethics Committee of Shanghai Children's Medical Center (approval number: SCMCIRB-Y2020100) prior 
to the data collection. Since the data were de-identified and aggregated, written consent was waived.

\section{Results}

There were 16,249 ARD cases in total, including 8179 HDM-ARD and 8070 NHDM-ARD, of which there were 11,437 outpatients and 4812 inpatients from 1 Jan 2013 to 31 Dec 2017. HDM-ARD accounted for more than one half of ARD population (50.3\%), and NHDM-ARD accounted for $49.7 \%$. The number of outpatient visits was far greater than inpatient visits for either of childhood ARD, HDM-ARD and NHDM-ARD. The average age of daily clinical visits for childhood ARD, HDM-ARD and NHDM-ARD was 4.8, 5.6 and 3.9 years, respectively, in which boys made up the majority $(62.7 \%, 64.0 \%, 61.4 \%$, respectively). The median and IQR of TIgE value for childhood ARD, HDM-ARD and NHDM-ARD was 123.0 (48.4 278), 235.0 (118 574) and 77.1 (26.3 146) IU/ ML, respectively (Table 1 ).

Additional file 1: Table S1 shows the summary statistics of air pollutants and daily clinical visits for childhood ARD, HDM-ARD and NHDM-ARD from 2013 to 2017. The median and IQR value of $\mathrm{NO}_{2}, \mathrm{SO}_{2}, \mathrm{PM}_{10}, \mathrm{PM}_{2.5}$, and $\mathrm{O}_{3}$ was $41(30 \sim 56) \mu \mathrm{g} / \mathrm{m}^{3}, 13(10 \sim 19) \mu \mathrm{g} / \mathrm{m}^{3}, 56$ $(40 \sim 82) \mu \mathrm{g} / \mathrm{m}^{3}, 41(26 \sim 63) \mu \mathrm{g} / \mathrm{m}^{3}$ and 72 (51 91.6) $\mu \mathrm{g} /$ $\mathrm{m}^{3}$, respectively. The daily median (IQR) number of clinical visits for childhood ARD, HDM-ARD and NHDMARD was 9 (5 13), $4(2 \sim 7)$ and $4(2 \sim 7)$, respectively. Additional file 1: Table S2 shows the associations of air pollutants and meteorological factor with childhood ARD, HDM-ARD and NHDM-ARD during 2013-2017. Spearman correlation coefficient between ARD and HDM-ARD was $0.88 \quad(\mathrm{p}<0.05)$. There were positive associations of $\mathrm{O}_{3}$ with HDM-ARD $\left(\mathrm{r}_{\mathrm{s}}=0.08, \mathrm{p}<0.05\right)$, while no association was found for other air pollutants. Additional file 1: Figs. S1, S2 and S3 depict a time series plot of apparent long-term trends and seasonality of clinical visits for childhood ARD, HDM-ARD and NHDMARD, respectively. Additional file 1: Fig. S4 indicates the distribution of air pollutants during the period.

Figure 2a shows that there was no statistically significant single-day effect of $\mathrm{O}_{3}$ on the daily clinical visits for childhood ARD. However, Fig. 2b reveals that higher $\mathrm{O}_{3}$ (relative risk (RR) $\operatorname{lag0-5}_{5}$ ) was significantly associated with an increased risk of clinical visits for ARD in children.

Figure 3a indicates that the strongest relationship between $\mathrm{O}_{3}$ and childhood HDM-ARD was found at lag 5 days. Figure $3 \mathrm{~b}$ depicts the cumulative lagged effects of $\mathrm{O}_{3}$ on the daily clinical visits for childhood HDM-ARD, suggesting that higher $\mathrm{O}_{3}\left(\mathrm{RR}_{\mathrm{lag} 0-1}\right.$ and $\left.\mathrm{RR}_{\mathrm{lag} 0-5}\right)$ was significantly associated with an increased risk of clinical visits for childhood HDM-ARD.

Figure $4 \mathrm{a}$ and $\mathrm{b}$ show the single-day effects and the cumulative lagged effects of $\mathrm{O}_{3}$ on the daily clinical visits for childhood NHDM-ARD, respectively. It indicates that $\mathrm{O}_{3}$ was not significantly associated with the risk of clinical visits for NHDM-ARD in children.

Additional file 1: Figs. S5 and S6 show the single-day effects and cumulative lagged effects of $\mathrm{NO}_{2}, \mathrm{SO}_{2}, \mathrm{PM}_{10}$ and $\mathrm{PM}_{2.5}$ on the daily clinical visits for ARD, HDM-ARD and NHDM-ARD in children, respectively. It suggested that $\mathrm{NO}_{2}, \mathrm{SO}_{2}, \mathrm{PM}_{10}$ and $\mathrm{PM}_{2.5}$ were not significantly associated with the risks of clinical visits for ARD, HDMARD and NHDM-ARD in children.

Table 2 shows the single-day effects and cumulative lagged effects of air pollutants on childhood ARD,

Table1 Characteristics of the patient episodes for ARD, HDM-ARD and NHDM-ARD

\begin{tabular}{|c|c|c|c|c|}
\hline variables & ARD & HDM-ARD & NHDM-ARD & p-value \\
\hline Total clinical visits & 16,249 & 8179 (50.3\%) & 8070 (49.7\%) & $<0.001$ \\
\hline Outpatient visits & $11,437(70.4 \%)$ & $6691(81.8 \%)$ & 4746 (58.8\%) & $<0.001$ \\
\hline Inpatient visits & $4812(29.6 \%)$ & $1488(18.2 \%)$ & $3324(41.2 \%)$ & $<0.001$ \\
\hline \multicolumn{5}{|l|}{ Age (years) } \\
\hline Mean (SD) & $4.8(2.8)$ & $5.6(2.8)$ & $3.9(2.5)$ & $<0.001$ \\
\hline$\leq 2$ & $2597(16.0 \%)$ & $436(5.3 \%)$ & $2161(26.8 \%)$ & $<0.001$ \\
\hline $3-6$ & $10,178(62.6 \%)$ & $5289(64.7 \%)$ & $4889(60.6 \%)$ & \\
\hline $7-17$ & 3474 (21.4\%) & $2454(30.0 \%)$ & $1020(12.6 \%)$ & \\
\hline \multicolumn{5}{|l|}{ Gender } \\
\hline Male & 10,193 (62.7\%) & $5238(64.0 \%)$ & 4955 (61.4\%) & $<0.001$ \\
\hline Female & $6056(37.3 \%)$ & 2941 (36.0\%) & $3115(38.6 \%)$ & \\
\hline \multicolumn{5}{|l|}{ TlgE (IU/ML) } \\
\hline Median, interquartile & $123.0(48.4 \sim 278)$ & $235.0(118 \sim 574)$ & $77.1(26.3 \sim 146)$ & $<0.001$ \\
\hline
\end{tabular}



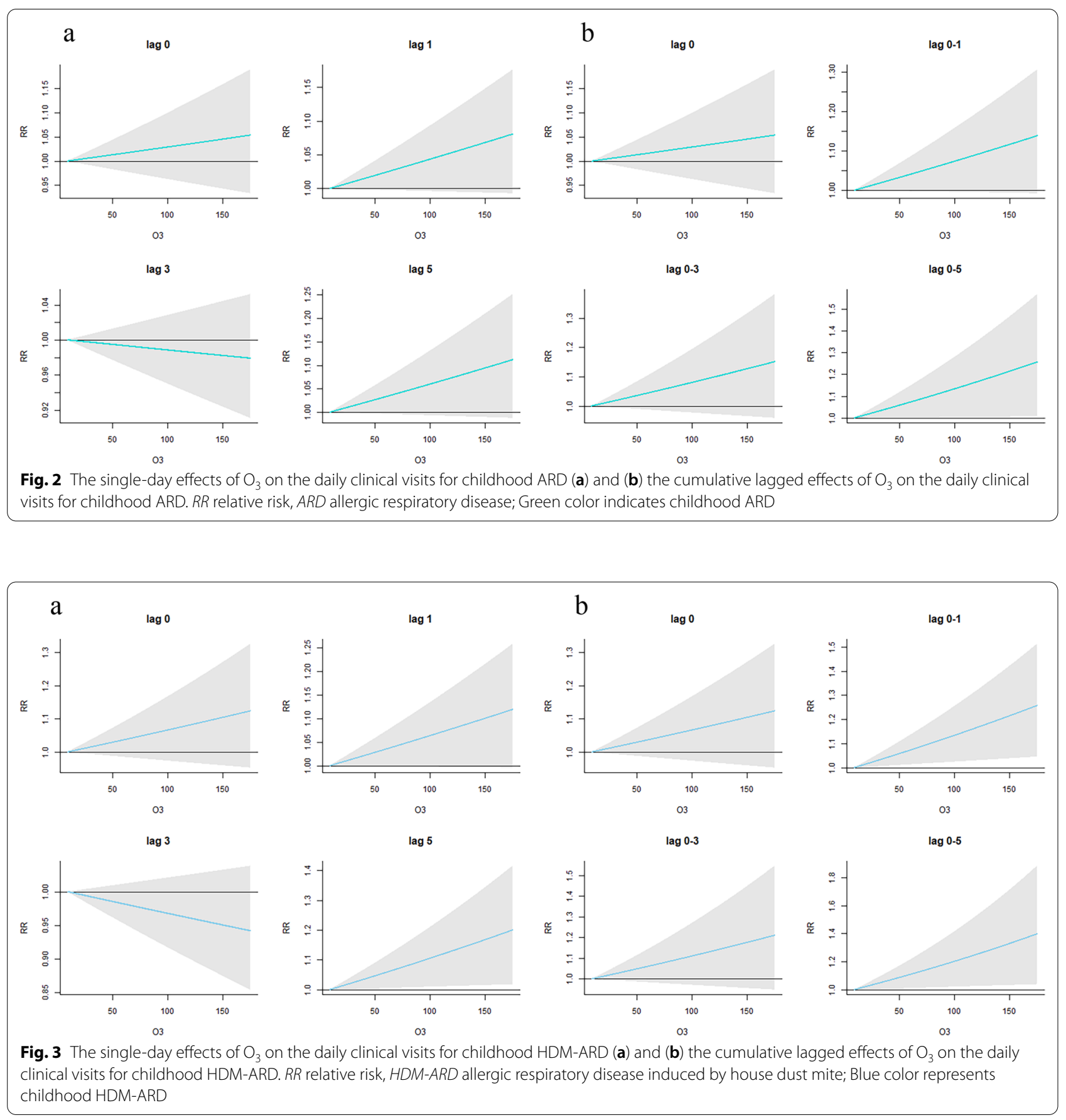

HDM-ARD and NHDM-ARD after taking putative confounders into account. The cut points of 95th percentile of $\mathrm{NO}_{2}, \mathrm{SO}_{2}, \mathrm{PM}_{10}, \mathrm{PM}_{2.5}$ and $\mathrm{O}_{3}$ were $87.0 \mu \mathrm{g} / \mathrm{m}^{3}$, $39.0 \mu \mathrm{g} / \mathrm{m}^{3}, 143.0 \mu \mathrm{g} / \mathrm{m}^{3}, 118.0 \mu \mathrm{g} / \mathrm{m}^{3}$, and $123.0 \mu \mathrm{g} / \mathrm{m}^{3}$, respectively. Exposure to higher $\mathrm{O}_{3}$ (95th percentile, $\left.123.0 \mu \mathrm{g} / \mathrm{m}^{3}\right)$ or an IQR increment $\left(40.6 \mu \mathrm{g} / \mathrm{m}^{3}\right)$ elevated the RR of childhood ARD $\left(\mathrm{RR}_{\mathrm{lag} 0-5}=1.19,95 \% \mathrm{CI}\right.$ : 1.03 , 1.38 , and $1.06,95 \%$ CI: $1.01,1.12$, respectively). However, the single-day effects of higher $\mathrm{O}_{3}$ and an IQR increment $\left(40.6 \mu \mathrm{g} / \mathrm{m}^{3}\right)$ were not significantly associated with the risk of clinical visits.

For HDM-ARD, there were stronger cumulative lagged effects of $\mathrm{O}_{3}$ exposure $\left(\mathrm{RR}_{\mathrm{lag} 0-5}\right.$ for the 95th percentile: $1.26,95 \% \mathrm{CI}: 1.03,1.55$; $\mathrm{RR}_{\mathrm{lag} 0-5}$ for IQR increment $\left.\left(40.6 \mu \mathrm{g} / \mathrm{m}^{3}\right): 1.09,95 \% \mathrm{CI}: 1.01,1.17\right)$ and the single-day effects $\left(R R_{\text {lag5 }}\right.$ for the 95 th percentile: 1.13 , 


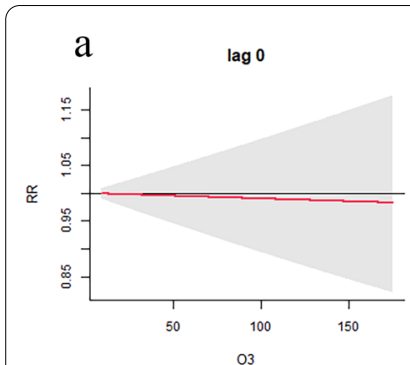

lag 3

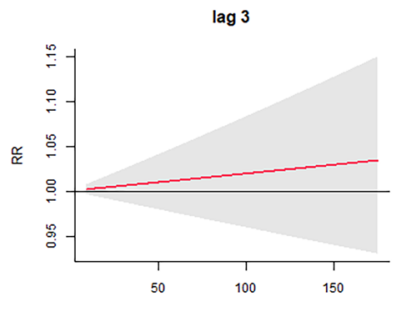

03

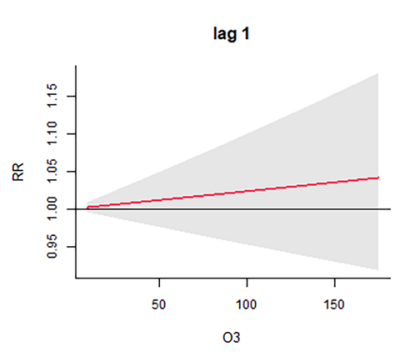

lag 5

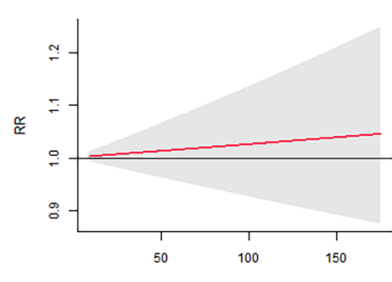

03

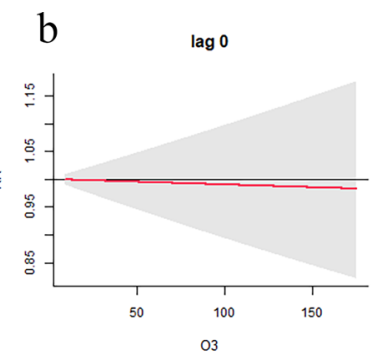

lag 0-3

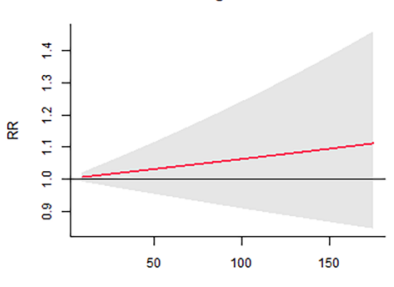

03

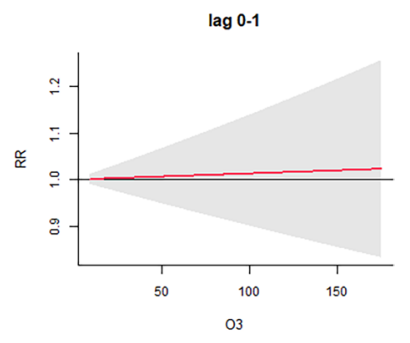

lag 0-5

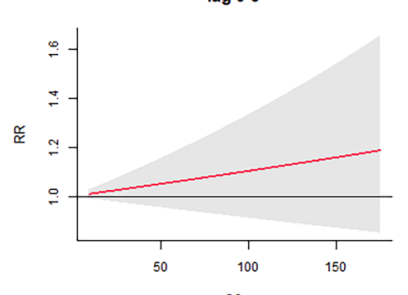

03

Fig. 4 The single-day effects of $\mathrm{O} 3$ on the daily clinical visits for childhood NHDM-ARD (a) and the cumulative lagged effects of $\mathrm{O}_{3}$ on the daily clinical visits for childhood NHDM-ARD (b). RR relative risk, NHDM-ARD allergic respiratory disease induced by non-house dust mite; Red color means childhood NHDM-ARD

95\% CI: $1.01,1.27 ; \mathrm{RR}_{\text {lag5 }}$ for IQR increment $(40.6 \mu \mathrm{g} /$ $\mathrm{m}^{3}$ ): 1.05, 95\% CI: 1.00, 1.09, respectively).

For NHDM-ARD, neither the single-day effects nor cumulative lagged effects of $\mathrm{O}_{3}$ exposure were significantly associated with the risk of clinical visits.

Figure 5 shows the results of the stratification analysis based on different genders in $\mathrm{O}_{3}$. Higher $\mathrm{O}_{3}$ was associated with increased RR of boys with ARD ( $R_{\text {lag0-5 }}$ for the 95th percentile: $1.26,95 \% \mathrm{CI}: 1.05,1.51$; $\mathrm{RR}_{\text {lag0-5 }}$ for IQR increment $\left.\left(40.6 \mu \mathrm{g} / \mathrm{m}^{3}\right): 1.09,95 \% \mathrm{CI}: 1.02,1.16\right)$ and HDM-ARD ( $\mathrm{RR}_{\text {lag0-5 }}$ for the 95th percentile: $1.36,95 \% \mathrm{CI}$ : 1.06, 1.75; $\mathrm{RR}_{\mathrm{lag} 0-5}$ for IQR increment $\left(40.6 \mu \mathrm{g} / \mathrm{m}^{3}\right): 1.11$, $95 \%$ CI: 1.02, 1.22), but not in girls. Additional file 1: Fig. S7 shows the results of subgroup analysis by gender in other pollutants. But there was no significant association for other air pollutants.

Additional file 1: Fig. S8 shows the overall exposureresponse relationships between exposure to $\mathrm{O}_{3}$ and clinical visits including outpatient and inpatient visits for ARD, HDM-ARD and NHDM-ARD. Additional file 1: Fig. S9 shows the overall exposure-response relationships between exposure to $\mathrm{O}_{3}$ and outpatient visits for ARD, HDM-ARD and NHDM-ARD. Additional file 1: Fig. S10 shows the overall exposure-response relationships between exposure to $\mathrm{O}_{3}$ and inpatient visits for ARD, HDM-ARD and NHDM-ARD. In the stratified analysis, for ARD and HDM-ARD, we found that $\mathrm{O}_{3}$ exposure was significantly associated with the increased risk of outpatient visits, while there was no such association for inpatient visits. There was no significant association between other air pollutants and inpatient or outpatient visits. In addition, the results show that both the single-day and cumulative exposures to $\mathrm{O}_{3}$ were significantly associated with the risk of clinical visits for the childhood HDM-ARD, whereas no such effect was found for childhood NHDM-ARD.

\section{Discussion}

To the best of our knowledge, this is the first time-series study to examine the relationship between air pollution and childhood ARD induced by specific allergen such as HDM. The key findings of this study include: (a) $\mathrm{O}_{3}$ exposure was significantly associated with clinical visits for childhood ARD; (b) there was a stronger relationship between $\mathrm{O}_{3}$ exposure and clinical visits for childhood HDM-ARD; (c) the effects of $\mathrm{O}_{3}$ exposure on childhood ARD and HDM-ARD were markedly lagged; (d) in stratified analyses, a significant association was only found for outpatient visits but not for inpatient visits.

High level of $\mathrm{O}_{3}$ was associated with the risk of clinical visits for childhood ARD, particularly for HDM-ARD. Therefore, exposure to $\mathrm{O}_{3}$, might significantly increase the exacerbation of HDM-ARD in children and threaten their respiratory health. Air pollutants lead to increased mucosal permeability through airway inflammation in susceptible subjects, promoting inhaled allergen penetration and entry into immune system [22]. The role of pollutants in increasing ARD sensitization and symptoms has been reviewed elsewhere [23-27]. HDM-an important factor leading to ARD, is the main inhaled allergen in Shanghai [28]. Ye et al. found that haze facilitates sensitization to HDM in children [29]. A cohort study in 


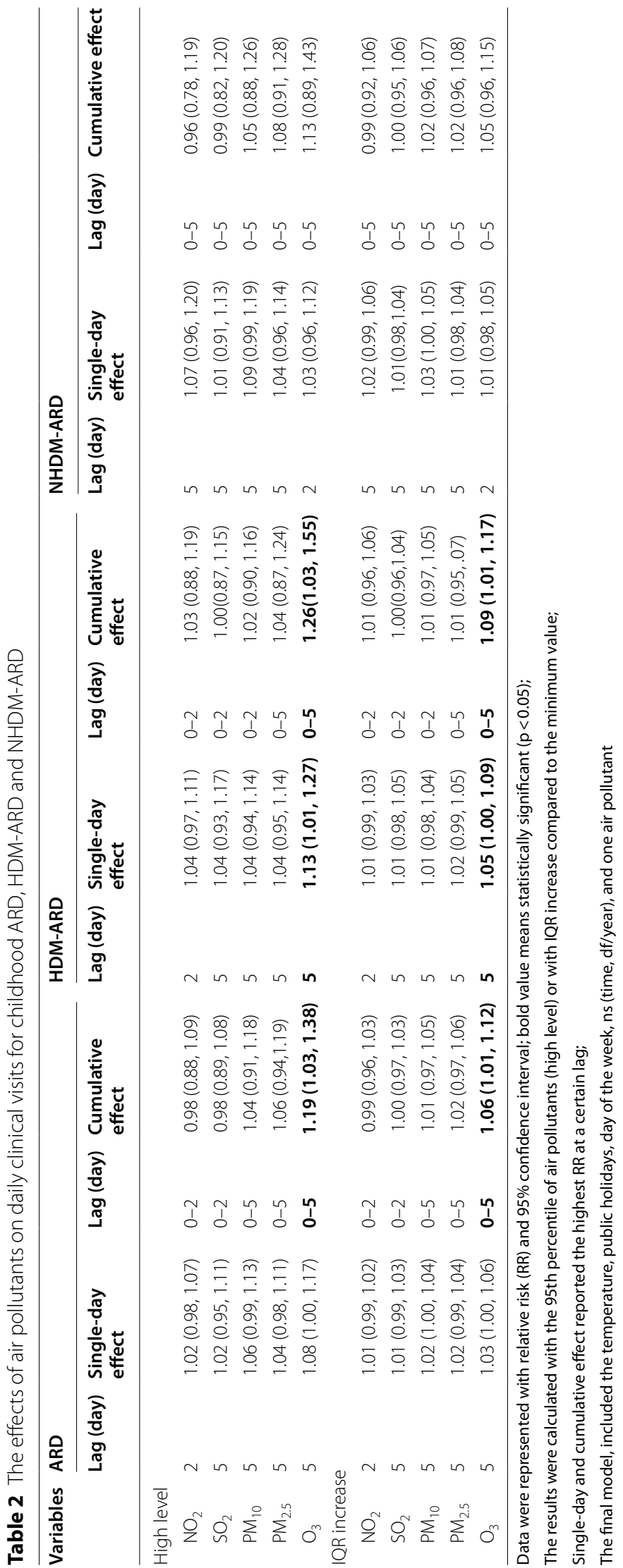




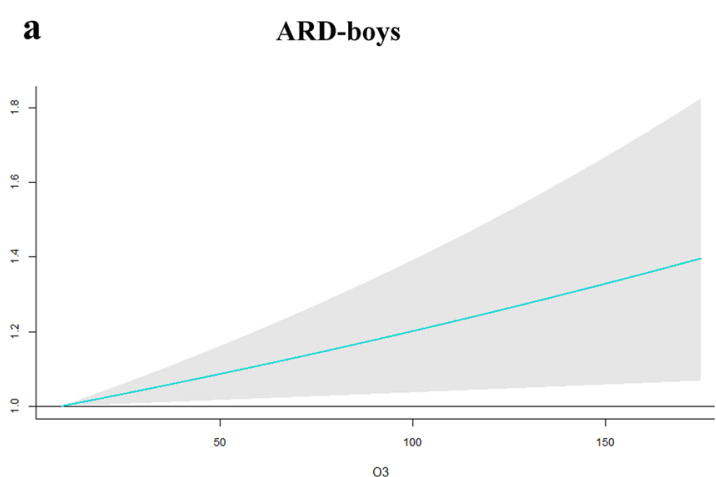

c

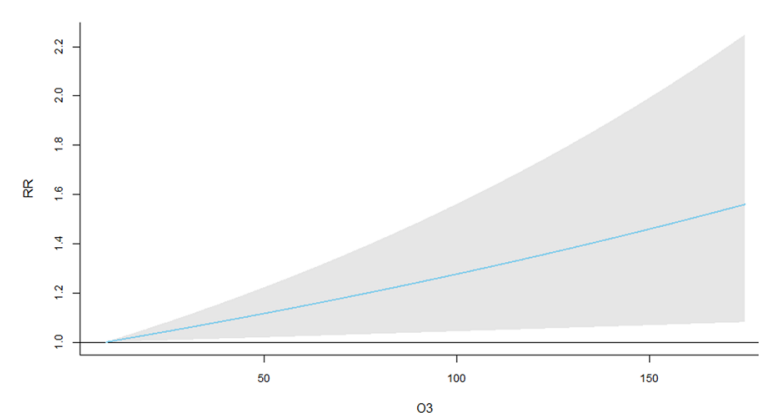

b

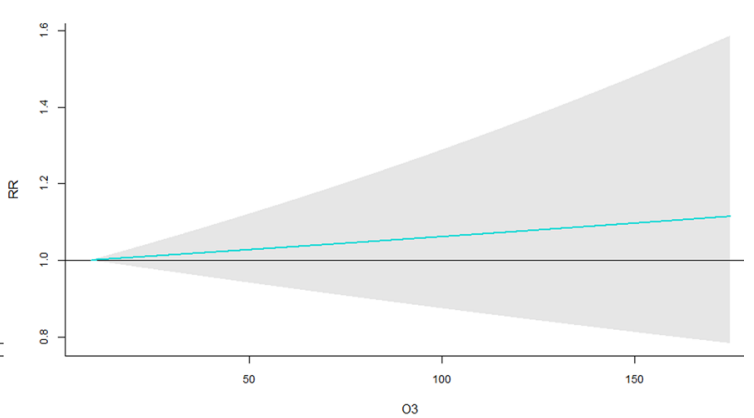

d

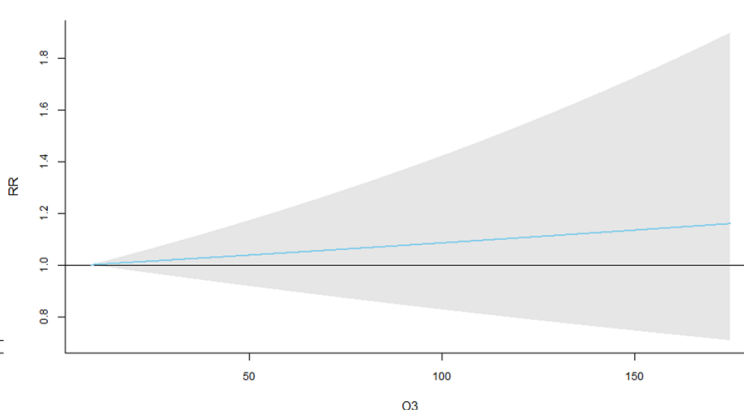

Fig. 5 The overall effects of $\mathrm{O}_{3}$ on clinical visits of childhood ARD based on different genders $(\mathbf{a}, \mathbf{b})$. The overall effects of $\mathrm{O}_{3}$ on clinical visits of childhood HDM-ARD based on different genders $(\mathbf{c}, \mathbf{d})$. RR relative risk, $A R D$ allergic respiratory disease, HDM-ARD allergic respiratory disease induced by house dust mite; Green color indicates childhood ARD; Blue color represents childhood HDM-ARD

Taiwan reported that children sensitized to HDM were most vulnerable to the adverse effects of air pollutants. In addition, HDM allergens may also alter the effects of air pollutants on ARD [9].

Although the mechanism underlying the relationship between air pollution and HDM-ARD was unclear, a review of mouse models and human studies suggests that the association might be mediated by an immune response [30]. Exposure high levels of air pollutants, particularly $\mathrm{O}_{3}, \mathrm{PM}, \mathrm{NO}_{2}$ and diesel exhaust, could alter innate immunity. There is also evidence that components of air pollutants, particularly $\mathrm{O}_{3}$, diesel exhaust particles and total PM, interact with allergens in the air [31]. Due to this interaction, air pollutants can promote lung penetration of aeroallergens by increasing the release of allergenic proteins, leading to allergenic sensitization, and promoting Th2 inflammation and allergen-specific IgE response. Other studies have shown that HDM can directly or indirectly activate airway epithelial cells, leading to a variety of changes in allergic airway inflammation and the occurrence of HDM-ARD [32]. Epigenetic modifications induced by HDM reveal several changes in bronchial tissue that lead to inflammation and bronchial hyperresponsiveness. Furthermore, epigenome might influence susceptibility to mite sensitization by hypomethylation of the IL13 gene and DNA methylation in B-cell [33, 34].

In this study, we conducted stratified analyses to examine the effects of air pollutants on outpatient and inpatient visits for childhood ARD, HDM-ARD and NHDM-ARD. The results show that for ARD and HDMARD, we found that $\mathrm{O}_{3}$ exposure was significantly associated with the increased risk of outpatient visits, but no such association was observed for inpatient visits. No significant association was found for other air pollutants. The previous studies have not yielded consistent results on associations between $\mathrm{O}_{3}$ exposure and clinical visits or hospital admissions for ARD. For example, $\mathrm{O}_{3}$ exposure exacerbated asthma and increased the risk of asthma emergency department visits in the Seattle area [35]. However, a study in Taiwan found no association between $\mathrm{O}_{3}$ exposure and daily hospital admissions for respiratory conditions [9].

According to the stratification analysis of different gender, we found that gender was the factor influencing the correlation between air pollutants and ARD and HDM-ARD. Higher $\mathrm{O}_{3}$ was associated with increased RR of male children with ARD and HDM-ARD, but not in female children. Several studies have shown that the airways of male and female children respond differently 
to air pollutants $[36,37]$. This is reasonable as there are differences in the airway between male and female children in the early and whole life stages of fetal lung development [38]. In childhood, the hyper-responsiveness of airway and ARD is more common among boys than girls [39]. As shown in this study, among the children, the stronger association between ambient $\mathrm{O}_{3}$ exposure and ARD and HDM-ARD was observed in males, which may be related to having less mature lungs and relatively narrower airways in boys than girls during childhood.

This study has four major strengths. First, this is the first time-series study to investigate the independent effects of air pollutants on childhood ARD, HDMARD and NHDM-ARD. Well-designed panel studies of time-series manner conducted in high risk (specific allergen sensitized) individuals could be sufficiently powered. Consistent with our findings, a cohort study on childhood environment and allergic diseases in Taiwan reported that children sensitized to HDM were most vulnerable to the adverse effects of air pollutants [9]. In addition, a large-scale cross-sectional study by Chen et al. found that $\mathrm{O}_{3}$ exposure may increase asthma exacerbation frequency [40]. Second, data from multi-sources including clinical records, air monitoring systems and meteorological services were integrated. Third, an advanced time series regression model (DLNM) was used in this study. The DLNM has increasingly been used in environmental health and epidemiological research. Finally, both single-day and cumulative effect estimates over 5 years were calculated to minimize short-term random variations.

Limitations of this study should also be acknowledged. Firstly, the cases in our study were selected from one hospital, and its generalizability may be limited. The findings of this study need to be interpreted with caution and multicenter studies are needed to validate these findings. Secondly, like other ecological time series studies, measurement bias is inevitable to some extent, since air pollution data were derived from monitoring stations, which could not be fully representative of individual exposures [41-43]. However, this type of measurement error may be non-differential, which may bias effect estimates towards the null [44]. Thirdly, some potential confounders that could affect the relationship between air pollution and ARD, such as influenza infections and life events [45], were not controlled for in this study because these data were unavailable.

To address the issues illustrated above, future research may focus on the following directions:

i. Prospective cohort studies are required to examine the causal/temporal relationship between environ- mental factors and childhood ARD, particularly HDM-ARD;

ii. Multi-center studies are needed to identify the influence of environmental factors on childhood ARD, HDM-ARD and NHDM-ARD;

iii. It is desirable to examine the interactive effects between air pollutants and meteorological factors on childhood ARD, HDM-ARD and NHDM-ARD;

iv. All potential confounding factors, including influenza infections and life events should be considered in further research.

\section{Conclusions}

$\mathrm{O}_{3}$ exposure was significantly associated with the increase of clinical visits for childhood ARD, especially for HDM-ARD. These findings contribute to an in-depth understanding of the etiology of HDM-ARD, and suggest that it may be beneficial to adopt control measures (e.g., increased ventilation and mite removal) to avoid co-exposure to allergens and air pollutants. Moreover, these findings shed light on the impacts of air pollution on ARD, HDM-ARD and NHDM-ARD, which may have significant ramifications for designing effective intervention programs to control and prevent childhood ARD, especially HDM-ARD, in China and other developing countries around the world.

\section{Abbreviations}

ARD: Allergic respiratory disease; HDM: House dust mite; HDM-ARD: Allergic respiratory disease induced by house dust mite; NHDM-ARD: Allergic respiratory disease induced by non-house dust mite; IQR: Interquartile range; ARIA: Allergic rhinitis and its impact on asthma; AR: Allergic rhinitis; AA: Allergic asthma; Der $p$ 1: Dermatophagoides pteronyssinus; Der $\mathrm{f} 1$ : Dermatophagoides farinae; TlgE: Total IgE; SlgE: Serum IgE; $\mathrm{NO}_{2}$ : Nitrogen dioxide; $\mathrm{SO}_{2}$ : Sulfur dioxide; $\mathrm{O}_{3}$ : Ozone; $\mathrm{PM}_{2.5}$ : Airborne particulate matter with an aerodynamic diameter less than $2.5 \mu \mathrm{m} ; \mathrm{PM}_{10}$ : Airborne particulate matter with an aerodynamic diameter less than $10 \mu \mathrm{m}$; Tmean: Daily mean temperature; SD: Standard deviation; DLNM: Distributed lag non-linear model; AIC: Akaike information criterion.

\section{Supplementary Information}

The online version contains supplementary material available at https://doi. org/10.1186/s12931-022-01967-1.

\footnotetext{
Additional file 1: Figure S1. Time-series plots of clinical visits for childhood ARD for seasonality and trend from 2013 to 2017. Figure S2. Timeseries plots of clinical visits for childhood HDM-ARD for seasonality and trend from 2013 to 2017. Figure S3. Time-series plots of clinical visits for childhood NHDM-ARD for seasonality and trend from 2013 to 2017. Figure S4. The distribution of air pollutants from 2013 to 2017. NO2: nitrogen dioxide; PM2.5: particulate matter less than $2.5 \mu \mathrm{m}$ in aerodynamic diameter; O3: ozone; SO2: sulfur dioxide; PM10: particulate matter less than $10 \mu \mathrm{m}$ in aerodynamic diameter. Blue smoothed lines were superimposed on each graph to present the long-term trends. Figure S5. The overall exposure-response association between NO2, SO2, PM10, PM2.5 and daily clinical visits for the single-day effects of childhood ARD, HDM-ARD and NHDM-ARD. RR: relative risk; ARD: allergic respiratory disease; HDM-ARD:
} 
allergic respiratory disease induced by house dust mite; NHDM-ARD: allergic respiratory disease induced by non-house dust mite; Green, blue and red color indicate childhood ARD, HDM-ARD, and NHDM-ARD, respectively. Figure S6. The overall exposure-response association between $\mathrm{NO} 2, \mathrm{SO} 2, \mathrm{PM} 10, \mathrm{PM} 2.5$ and daily clinical visits for the cumulative lagged effects of childhood ARD, HDM-ARD and NHDM-ARD. RR: relative risk; ARD: allergic respiratory disease; HDM-ARD: allergic respiratory disease induced by house dust mite; NHDM-ARD: allergic respiratory disease induced by non-house dust mite; Green, blue and red color indicate childhood ARD, HDM-ARD, and NHDM-ARD, respectively. Figure S7. The overall exposureresponse association between NO2, SO2, PM10, PM2.5 and daily clinical visits for childhood ARD and HDM-ARD based on different genders. RR: relative risk; ARD: allergic respiratory disease; HDM-ARD: allergic respiratory disease induced by house dust mite; Green color indicates childhood ARD; Blue color represents childhood HDM-ARD. Figure S8. The overall exposure-response relationships between air pollutants and clinical visits for childhood ARD, HDM-ARD and NHDM-ARD. RR: relative risk; ARD: allergic respiratory disease; HDM-ARD: allergic respiratory disease induced by house dust mite; NHDM-ARD: allergic respiratory disease induced by non-house dust mite; Green, blue and red color indicate childhood ARD, HDM-ARD, and NHDM-ARD, respectively. Figure S9. The overall exposure-response relationships between air pollutants and outpatient visits for childhood ARD, HDM-ARD and NHDM-ARD. RR: relative risk; ARD: allergic respiratory disease; HDM-ARD: allergic respiratory disease induced by house dust mite; NHDM-ARD: allergic respiratory disease induced by non-house dust mite; Green, blue and red color indicate childhood ARD, HDM-ARD, and NHDM-ARD, respectively. Figure S10. The overall exposure-response relationships between air pollutants and inpatient visits for childhood ARD, HDM-ARD and NHDM-ARD. RR: relative risk; ARD: allergic respiratory disease; HDM-ARD: allergic respiratory disease induced by house dust mite; NHDM-ARD: allergic respiratory disease induced by non-house dust mite; Green, blue and red color indicate childhood ARD, HDM-ARD, and NHDM-ARD, respectively. Table S1. Distribution of daily clinical visits for childhood ARD, HDM-ARD, NHDM-ARD and air pollutants from 2013 to 2017. Table S2. Spearman correlation coefficients between environmental factors during 2013-2017.

\section{Acknowledgements}

We would like to thank Shanghai Children's Medical Center, Shanghai Meteorological Center and Shanghai Environmental Protection Agency for providing hospital and environmental data.

\section{Authors' contributions}

YL and ST were responsible for the conception and design of the study. YH carried out the statistical analysis. JL wrote the initial draft of the manuscript. All authors (1) provided substantial contributions to the conception or design of the work, or the acquisition, analysis or interpretation of the data for the work, (2) revised the manuscript critically for important intellectual content and (3) approved the final version for submission. All authors read and approved the final manuscript.

\section{Funding}

This study was supported by a Grant from Chinese National Natural Science Foundation [82071015], and a Grant from the Science and Technology Commission of Shanghai Municipality [21Y11900200] and [18411951600].

\section{Availability of data and materials}

The data that support the findings in this study are available from the corresponding author upon reasonable request.

\section{Declarations}

\section{Ethics approval and consent to participate}

The ethical approval of this project was Granted by the Ethics Committee of Shanghai Children's Medical Center (approval number: SCMCIRB-Y2020100) prior to the data collection. Since the data were de-identified and aggregated, written consent was not needed. No personal information was gathered throughout the study.

\section{Consent for publication}

Not applicable.

\section{Competing interests}

The authors declare that they have no conflicts of interest.

\section{Author details}

${ }^{1}$ Department of Otolaryngology, Shanghai Children's Medical Center, School of Medicine, Shanghai Jiao Tong University, 1678 Dongfang Rd, Pudong, Shanghai 200127, China. ${ }^{2}$ Department of Clinical Epidemiology and Biostatistics, Shanghai Children's Medical Center, School of Medicine, Shanghai Jiao Tong University, 1678 Dongfang Rd, Pudong, Shanghai 200127, China. ${ }^{3}$ Department of Clinical Laboratory Medicine, Shanghai Children's Medical Center, School of Medicine, Shanghai Jiao Tong University, Shanghai, China. ${ }^{4}$ School of Public Health, Institute of Environment and Population Health, Anhui Medical University, Hefei, China. ${ }^{5}$ Center for Global Health, School of Public Health, Nanjing Medical University, Nanjing, China. ${ }^{6}$ School of Public Health and Social Work, Queensland University of Technology, Brisbane, Australia.

Received: 26 September 2021 Accepted: 21 February 2022

Published online: 05 March 2022

\section{References}

1. Bousquet J, Van Cauwenberge P, Khaltaev N. Allergic rhinitis and its impact on asthma. J Allergy Clin Immunol. 2001;108:S147-334. https:// doi.org/10.1067/mai.2001.118891.

2. Savouré $M$, Bousquet J, Burte $E$, et al. Questionnaire as an alternative of skin prick tests to differentiate allergic from non-allergic rhinitis in epidemiological studies. Allergy. 2021. https://doi.org/10.1111/all.14812.

3. Urrutia-Pereira M, Chong-Neto H, Mocellin LP, et al. Prevalence of asthma symptoms and associated factors in adolescents and adults in southern Brazil: a global asthma network phase I study. World Allergy Organ J. 2021;14: 100529. https://doi.org/10.1016/j.waojou.2021.100529.

4. Mikhail I, Stukus DR, Prince BT. Fatal anaphylaxis: epidemiology and risk factors. Curr Allergy Asthma Rep. 2021. https://doi.org/10.1007/ s11882-021-01006-X.

5. Naclerio R, Ansotegui IJ, Bousquet J, et al. International expert consensus on the management of allergic rhinitis (AR) aggravated by air pollutants: Impact of air pollution on patients with AR: current knowledge and future strategies. World Allergy Organ J. 2020;13: 100106. https://doi.org/ 10.1016/j.waojou.2020.100106.

6. Pawankar R, Wang JY. Climate change, air pollution, and biodiversity in Asia pacific and impact on respiratory allergies. Immunol Allergy Clin North Am. 2021;41:63-71. https://doi.org/10.1016/j.iac.2020.09.008.

7. Traina $G$, Barbalace A, Betti F, et al. What impact of air pollution in pediatric respiratory allergic diseases. Pediatr Allergy Immunol. 2020;31 (Suppl 26):26-8. https://doi.org/10.1111/pai.13362.

8. D'Amato G, Cecchi L, D'Amato M, Liccardi G. Urban air pollution and climate change as environmental risk factors of respiratory allergy: an update. J Investig Allergol Clin Immunol. 2010;20:95-102. https://doi.org/ 10.1016/j.jaci.2009.10.034.

9. Wang IJ, Tung TH, Tang CS, Zhao ZH. Allergens, air pollutants, and childhood allergic diseases. Int J Hyg Environ Health. 2016;219:66-71. https:// doi.org/10.1016/j.ijheh.2015.09.001.

10. Calderon MA, Kleine-Tebbe J, Linneberg A, et al. House dust mite respiratory allergy: an overview of current therapeutic strategies. J Allergy Clin Immunol Pract. 2015;3:843-55. https://doi.org/10.1016/j.jaip.2015.06.019.

11. Boulet LP, Gauvreau G, Boulay ME, O'Byrne PM, Cockcroft DW. Allergeninduced early and late asthmatic responses to inhaled seasonal and perennial allergens. Clin Exp Allergy. 2015;45:1647-53. https://doi.org/10. 1111/cea.12587.

12. Koehler C, Paulus M, Ginzkey C, et al. The proinflammatory potential of nitrogen dioxide and its influence on the house dust mite allergen Der p 1. Int Arch Allergy Immunol. 2016;171:27-35. https://doi.org/10.1159/ 000450751. 
13. Winter WE, Hardt NS, Fuhrman S. Immunoglobulin E: importance in parasitic infections and hypersensitivity responses. Arch Pathol Lab Med. 2000;124:1382-5. https://doi.org/10.5858/2000-124-1382-IE.

14. Li Y, Zhu L, Chen J, et al. Perioperative levels of total IgE correlate with outcomes of prolonged mechanical ventilation after cardiopulmonary bypass in pediatric patients. Pediatr Res. 2018;84:689-95. https://doi.org/ 10.1038/s41390-018-0048-2.

15. Li Y, Chen J, Rui X, et al. The association between sixteen genome-wide association studies-related allergic diseases loci and childhood allergic rhinitis in a Chinese Han population. Cytokine. 2018;111:162-70. https:// doi.org/10.1016/j.cyto.2018.08.022.

16. Hu Y, Cheng J, Jiang F, et al. Season-stratified effects of meteorological factors on childhood asthma in Shanghai. China Environ Res. 2020;191: 110115. https://doi.org/10.1016/j.envres.2020.110115.

17. Wei Q, Zhong L, Gao J, et al. Diurnal temperature range and childhood asthma in Hefei, China: does temperature modify the association? Sci Total Environ. 2020;724: 138206. https://doi.org/10.1016/j.scitotenv.2020. 138206.

18. Gasparrini A, Armstrong B, Kenward MG. Distributed lag non-linear models. Stat Med. 2010;29:2224-34. https://doi.org/10.1002/sim.3940.

19. Chen K, Glonek G, Hansen A, et al. The effects of air pollution on asthma hospital admissions in Adelaide, South Australia, 2003-2013: time-series and case-crossover analyses. Clin Exp Allergy. 2016;46:1416-30. https:// doi.org/10.1111/cea.12795.

20. Xu Z, Huang C, Su H, et al. Diurnal temperature range and childhood asthma: a time-series study. Environ Health. 2013;12:12. https://doi.org/ 10.1186/1476-069x-12-12.

21. Yi W, Chan AP. Effects of temperature on mortality in Hong Kong: a time series analysis. Int J Biometeorol. 2015;59:927-36. https://doi.org/10.1007/ s00484-014-0895-4.

22. D'Amato G. Effects of climatic changes and urban air pollution on the rising trends of respiratory allergy and asthma. Multidiscip Respir Med. 2011;6:28-37. https://doi.org/10.1186/2049-6958-6-1-28.

23. Burbank AJ, Sood AK, Kesic MJ, Peden DB, Hernandez ML. Environmental determinants of allergy and asthma in early life. J Allergy Clin Immunol. 2017;140:1-12. https://doi.org/10.1016/j.jaci.2017.05.010.

24. Norbäck D, Lu C, Zhang Y, et al. Sources of indoor particulate matter (PM) and outdoor air pollution in China in relation to asthma, wheeze rhinitis and eczema among pre-school children: synergistic effects between antibiotics use and PM(10) and second hand smoke. Environ Int. 2019;125:252-60. https://doi.org/10.1016/j.envint.2019.01.036.

25. Kim KH, Jahan SA, Kabir E. A review on human health perspective of air pollution with respect to allergies and asthma. Environ Int. 2013;59:41-52. https://doi.org/10.1016/j.envint.2013.05.007.

26. Khreis H, Kelly C, Tate J, et al. Exposure to traffic-related air pollution and risk of development of childhood asthma: a systematic review and metaanalysis. Environ Int. 2017;100:1-31. https://doi.org/10.1016/j.envint.2016. 11.012.

27. Zheng $X Y$, Ding $H$, Jiang $L N$, et al. Association between Air Pollutants and Asthma Emergency Room Visits and Hospital admissions in time series studies: a systematic review and meta-analysis. PLoS ONE. 2015;10: e0138146. https://doi.org/10.1371/journal.pone.0138146.

28. Ding JJ, Zhang JH, Xu F, Xu Y, Zhu HB. Analyzing of the inhaled allergens profiles of 890 allergic rhinitis patients. Lin Chung Er Bi Yan Hou Tou Jing Wai Ke Za Zhi. 2012;26:164-6. https://doi.org/10.13201/j.issn.1001-1781. 2012.04.014.

29. Ye Q, Zhang T, Mao JH. Haze facilitates sensitization to house dust mites in children. Environ Geochem Health. 2020;42:2195-203. https://doi.org/ 10.1007/s10653-019-00481-6.

30. Miller RL, Peden DB. Environmental effects on immune responses in patients with atopy and asthma. J Allergy Clin Immunol. 2014;134:10018. https://doi.org/10.1016/j.jaci.2014.07.064.

31. Gandhi VD, Vliagoftis H. Airway epithelium interactions with aeroallergens: role of secreted cytokines and chemokines in innate immunity. Front Immunol. 2015;6:147. https://doi.org/10.3389/fimmu.2015.00147.

32. Gandhi VD, Davidson C, Asaduzzaman M, Nahirney D, Vliagoftis H. House dust mite interactions with airway epithelium: role in allergic airway inflammation. Curr Allergy Asthma Rep. 2013;13:262-70. https://doi.org/ 10.1007/s11882-013-0349-9.
33. Pascual M, Suzuki M, Isidoro-Garcia M, et al. Epigenetic changes in B lymphocytes associated with house dust mite allergic asthma. Epigenetics. 2011;6:1131-7. https://doi.org/10.4161/epi.6.9.16061.

34. Li JY, Zhang Y, Lin XP, et al. Association between DNA hypomethylation at IL13 gene and allergic rhinitis in house dust mite-sensitized subjects. Clin Exp Allergy. 2016;46:298-307. https://doi.org/10.1111/cea.12647.

35. Mar TF, Koenig JQ. Relationship between visits to emergency departments for asthma and ozone exposure in greater Seattle. Washington Ann Allergy Asthma Immunol. 2009;103:474-9. https://doi.org/10.1016/ s1081-1206(10)60263-3.

36. Rojas-Martinez R, Perez-Padilla R, Olaiz-Fernandez G, et al. Lung function growth in children with long-term exposure to air pollutants in Mexico City. Am J Respir Crit Care Med. 2007;176:377-84. https://doi.org/10. 1164/rccm.200510-16780C.

37. Gauderman WJ, Vora H, McConnell R, et al. Effect of exposure to traffic on lung development from 10 to 18 years of age: a cohort study. Lancet. 2007;369:571-7. https://doi.org/10.1016/s0140-6736(07)60037-3.

38. Becklake MR, Kauffmann F. Gender differences in airway behaviour over the human life span. Thorax. 1999;54:1119-38. https://doi.org/10.1136/ thx.54.12.1119.

39. Dong GH, Chen T, Liu MM, et al. Gender differences and effect of air pollution on asthma in children with and without allergic predisposition: northeast Chinese children health study. PLoS ONE. 2011;6: e22470. https://doi.org/10.1371/journal.pone.0022470.

40. Chen CH, Chan CC, Chen BY, Cheng TJ, Leon GY. Effects of particulate air pollution and ozone on lung function in non-asthmatic children. Environ Res. 2015;137:40-8. https://doi.org/10.1016/j.envres.2014.11.021.

41. Tian L, Liang F, Guo Q, et al. The effects of interaction between particulate matter and temperature on mortality in Beijing. China Environ Sci Process Impacts. 2018;20:395-405. https://doi.org/10.1039/c7em00414a.

42. Weiss SC, Rowell R, Krochmal L. Impact of seasonality on conducting clinical studies in dermatology. Clin Dermatol. 2008;26:565-9. https://doi. org/10.1016/j.clindermatol.2008.01.016.

43. Denda M, Sokabe T, Fukumi-Tominaga T, Tominaga M. Effects of skin surface temperature on epidermal permeability barrier homeostasis. J Invest Dermatol. 2007;127:654-9. https://doi.org/10.1038/sj.jid.5700590.

44. Brenner H, Loomis D. Varied forms of bias due to nondifferential error in measuring exposure. Epidemiology. 1994;5:510-7. https://doi.org/10. 2307/3432139.

45. Caraballo L, Acevedo N, Zakzuk J. Ascariasis as a model to study the helminth/allergy relationships. Parasite Immunol. 2019;41: e12595. https:// doi.org/10.1111/pim.12595.

\section{Publisher's Note}

Springer Nature remains neutral with regard to jurisdictional claims in published maps and institutional affiliations.

Ready to submit your research? Choose BMC and benefit from:

- fast, convenient online submission

- thorough peer review by experienced researchers in your field

- rapid publication on acceptance

- support for research data, including large and complex data types

- gold Open Access which fosters wider collaboration and increased citations

- maximum visibility for your research: over 100M website views per year

At BMC, research is always in progress.

Learn more biomedcentral.com/submissions 